\title{
有机合成中腈的去对称化生物转化反应研究进展
}

\author{
敖宇飞*,a,b 王其强 ${ }^{a}$ 王德先 ${ }^{a, b}$ \\ ( ${ }^{a}$ 中国科学院化学研究所 北京分子科学国家实验室 中国科学院分子识别与功能重点实验室 北京 100190) \\ $\left({ }^{b}\right.$ 中国科学院大学 北京 100049)
}

\begin{abstract}
摘要 腈的化学水合和水解具有反应条件苛刻、低选择性等缺点, 与之相反, 腈的去对称化生物转化反应具有反应条 件温和、高效、高选择性以及理论产率可达 $100 \%$ 的优点，已成为合成对映体纯多官能化羧酸和酰胺的最有效方法之一. 将分别从前手性戍二腈、前手性丙二腈、内消旋环状二腈以及其他类底物出发, 简单综述腈类化合物的去对称化生物 转化反应及其在有机合成中的应用进展, 并对各类反应的条件、选择性以及规律机制进行讨论和总结.
\end{abstract}

关键词 去对称化; 生物转化; 二腈

\section{Biocatalytic Desymmetrization of Dinitriles in Organic Synthesis}

\author{
Ao, Yufei* ${ }^{*, a, b}$ Wang, Qiqiang ${ }^{a} \quad$ Wang, Dexian ${ }^{a, b}$ \\ ( ${ }^{a}$ Beijing National Laboratory for Molecular Sciences, CAS Key Laboratory of Molecular Recognition and \\ Function Institute of Chemistry, Chinese Academy of Sciences, Beijing 100190) \\ ( ${ }^{b}$ University of Chinese Academy of Sciences, Beijing 100049)
}

\begin{abstract}
In comparison with the chemical hydration and hydrolysis of nitriles, which usually involves harsh reaction conditions and low selectivity, biocatalytic desymmetrizations of prochiral or meso nitriles are highly efficient, highly enantioselective and environmentally benign. Therefore, biocatalysis and biotransformation has offered an attractive and unique protocol for the enantioselective synthesis of polyfunctionalized organic compounds that are not readily obtainable by other methods. This review summarizes the biocatalytic desymmetrization of prochiral nitriles including glutaronitriles, malonitriles and meso cyclic dinitriles during the past two decades.
\end{abstract}

Keywords desymmetrization; biotransformations; dinitriles

生物转化是利用生物催化体系(如各种细胞和酶等) 实现的反应过程, 它是迄今为止人类所知的最高效和最 具有选择性的温和催化反应体系，也是一个产生污染物 少、催化剂可降解的环境友好体系. 生物转化方法可以 获得很多常规化学方法不能或难以合成的高纯度手性 化合物. 近几十年来, 生物转化获得了蓬勃的发展, 现 在人们能够利用生物催化剂催化几乎所有种类的有机 化学反应, 其中由于生物催化水解反应不需要敏感的辅 酶且水解酶的底物范围较广, 因而相关的研究最多 ${ }^{[1]}$.

腈类化合物是一类重要的有机合成中间体 ${ }^{[2]}$, 它们 不仅可以通过多种途径容易地制备, 还可以转化成含有 不同官能团的化合物. 在实验室和工业界, 将腈进行化 学水合或化学水解可以实现酰胺和羧酸的合成, 但是,
因为这些反应通常需要较苛刻的条件(例如在强酸或强 碱条件下加热回流), 所以当目标产物分子中带有化学 敏感基团时, 往往需要进行额外的保护-去保护反应方 能实现其合成, 另外, 这类反应的化学、区域和立体选 择性也较差. 与之形成鲜明对比的是, 通过生物转化反 应, 腈可以在非常温和的条件下转化成相应的酰胺和羒 酸(例如在中性条件下 $30{ }^{\circ} \mathrm{C}$ 反应), 而且在反应中还能 体现优良的选择性 ${ }^{[3]}$. 自然界中, 腈的生物水解反应包 括两种途径: 一是在腈水解酶(nitrilase, E.C.3.5.5.1)的作 用下, 将腈直接水解成羧酸和氨气 ${ }^{[4]}$, 二是先经腈水合 酶(nitrile hydratase, E.C.4.2.1.84)的催化水合得到酰胺, 然后在酰胺水解酶(amidase, E.C.3.5.1.4)的催化下水解 得到羞酸 ${ }^{[5]}$ (Scheme 1).

\footnotetext{
*E-mail: aoyufe@iccas.ac.cn

Received May 9, 2016; revised June 11, 2016; published online June 20, 2016.

Project supported by the National Natural Science Foundation of China (No. 21502202).

国家自然科学基金(No. 21502202)资助项目.
} 


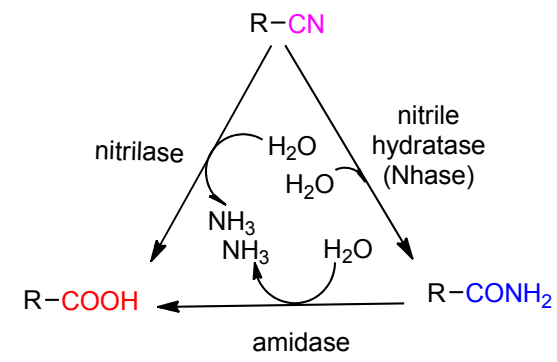

图式 1 腈类化合物的生物水解途径

Scheme 1 Two distinct pathways for nitrile biodegradation

人们根据酶的晶体结构推测相应的催化机制. Brenner ${ }^{[6 a]}$ 认为腈水解酶的 Glu-Lys-Cys 三个残基对催化 反应起了至关重要的作用, 其中半胱氨酸残基上的颈基 亲核进攻底物上的碳原子, 水分子参与反应并产生氨 气。 郑裕国课题组 ${ }^{[6 b]}$ 对菌株 Alcaligenes faecalis ZJUTB10 的腈水解酶进行了系统的研究, 给出了酶与 扁桃腈底物结合的中间体模型, 并通过计算解释了酶对 $R$-扁桃腈有更高的催化速率. 与腈水解酶不同, 腈水合 酶的催化活性部位一般含有一个非血红素铁(non-heme ion)或非类可啉钴(non-corrinoid colbalt ${ }^{[7 a]}$, 钱世钧等 ${ }^{[7 b]}$ 报道了 Rhodococcus erythropolis AJ270 的腈水合酶晶体 结构, 并推测其催化机理 (Scheme 2), 即氰基上的氮先 与铁配位处的水分子形成氢键削弱碳氮三键, 另一水分 子被谷氨酰胺残基的酰胺基团活化, 其氧原子对氧基亲 核进攻, 最终形成酰胺. 腈水合酶和酰胺水解酶的基因 往往存在于同一操纵子上, 因此它们往往是同时表达的 ${ }^{[8 a]}$, Ohtaki 等 ${ }^{[8 b]}$ 报道了 Rhodococcus sp. N-771 的酰胺水 解酶晶体结构, 发现 Ser-cis Ser-Lys 组成催化三联体, 其中丝氨酸残基上的羟基亲核进攻底物上的碳原子.

目前对腈类化合物的对映选择性生物水解反应的 研究主要采用动力学拆分的方法 ${ }^{[9]}$, 但其理论产率至高 为 $50 \%$, 与之相比, 去对称化反应可以得到 $100 \%$ 理论 产率的单一对映体产物, 因而具有更高的应用潜力 ${ }^{[10]}$. 腈的去对称化生物转化反应中涉及到两个氧基, 所以其 反应途径比单腈的更复杂(Scheme 3).
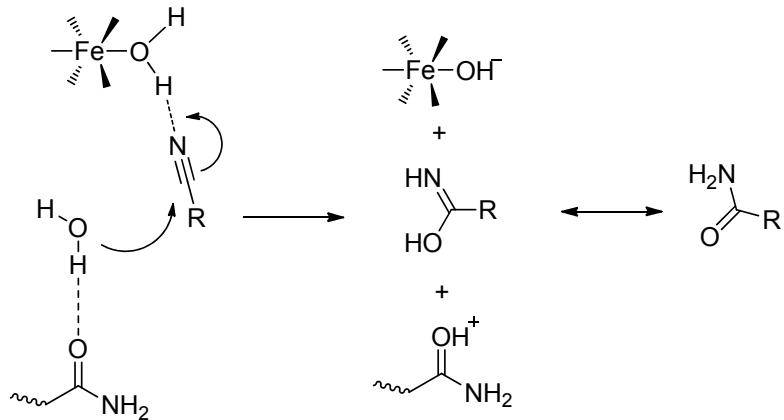

图式 2 Rhodococcus erythropolis AJ270 的腈水合酶的推测机 理

Scheme 2 Proposed mechanism of nitrile hydratase from Rhodococcus erythropolis AJ270

对于含腈水解酶的催化体系, 采用路径 A 模式, 即 底物 $\mathbf{a}$ 先在腈水解酶的作用下转化为单氰基羧酸 $\mathbf{c}$, 如 果酶的选择性足够好, 则反应停留在此阶段生成普通化 学水解方法难以得到的单氧基羧酸产物; 相反, 如果酶 的选择性不好, 且活性足够高, 则进一步转化为二羧酸 f. 对于含腈水合酶/酰胺水解酶的催化体系, 采用路径 $\mathrm{B}$ 模式, 底物 $\mathbf{a}$ 先在腈水合酶的作用下转化为单氰基酰 胺 $\mathbf{b}$, 随后可能发生两种反应，一是单氰基酰胺在酰胺 水解酶的作用下转化为单氧基羧酸 $\mathbf{c}$, 随后可能在腈水 合酶的作用下进一步转化为单酰胺羧酸 $\mathbf{e}$; 另一种是单 氧基酰胺 $\mathbf{b}$ 在腈水合酶的作用下继续转化为二酰胺 $\mathbf{d}$, 随后可能在酰胺水解酶的作用下进一步生成单酰胺羧 酸 $\mathbf{e}$, 如果酰胺水解酶的选择性不好, 且活性足够高, 单酰胺羧酸可以转化为二羧酸 f. 因为路径 B 涉及两种 酶的作用, 其产物组成往往比较复杂, 但是依靠控制反 应条件和反应时间的方法往往能够使反应停留在中间 某步, 且依靠酶的高对映选择性往往能够得到高光学纯 的单一产物. 此外人们常直接以二酰胺 $\mathbf{d}$ 为底物进行去 对称化生物转化研究, 此法只涉及酰胺水解酶的催化过 程，更容易控制反应条件实现单一产物 $\mathbf{e}$ 的获得.
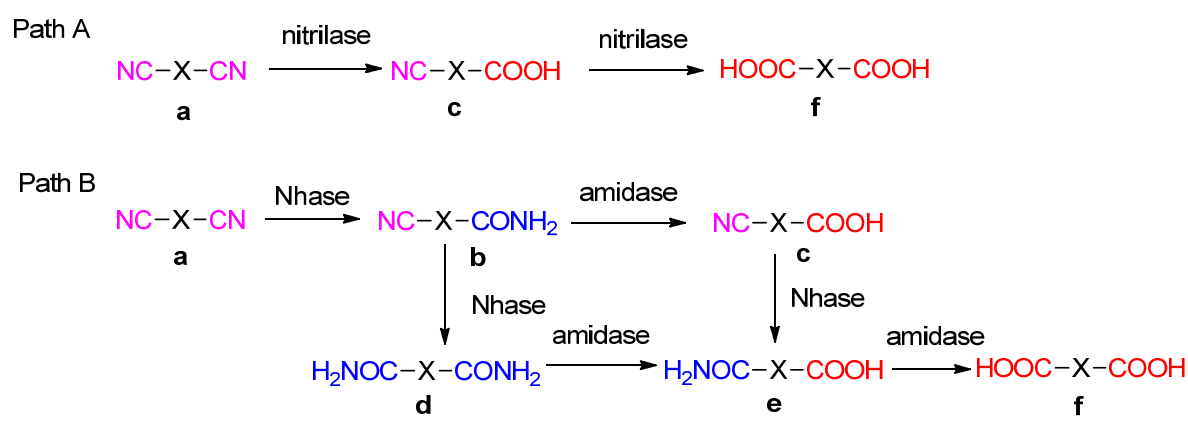

图式 3 二腈生物水解的可能途径

Scheme 3 Possible procedure of the hydrolysis of dinitriles 
生物转化反应的效率和对映选择性通常与底物的 结构有密切的联系, 因此, 本文从不同结构的底物出发, 分为四部分介绍腈类化合物的去对称化生物转化反应 并展示其在有机合成中的应用, 最后对这一领域给出总 结与展望.

\section{1 前手性戊二腈类底物的去对称化生物转化反} 应

3-取代戊二腈被选作去对称化生物转化反应的底 物, 是因为底物合成简单且其可能的水解产物是合成一 些手性药物的关键中间体, 因此对这类底物的研究开展 的最早, 相关报道较多.

1991 年, Ohta 课题组 ${ }^{[11]}$ 首次报道了 3-芐基、3-苄氧 基和 3-苯甲酰氧基戊二腈 $\mathbf{1}$ 在 Rhodococcus butanica ATCC 21197(该菌株后来更名为 Rhodococcus rhodochrous IFO 15564)整细胞催化下的去对称化生物转化反 应(Eq. 1), 反应可以在非常温和的条件下发生, 生成 $S$ 构型的单氰基单羒酸产物 2 , 特别是可以以较高产率得 到对映体纯的产物 S-3-苯甲酰氧基-4-氧基丁酸 (2c), 作 者认为底物上的苯环结构是获得手性产物的关键因素. 后续的研究表明, 该菌株含有腈水合酶和酰胺水解酶, 虽然在这篇报道中作者并没有对酶的效率和选择性进 行讨论, 但是从反应结果可以看出, 酶催化这类底物具 有较高的效率, 而酶的选择性与底物上取代基的结构密 切相关，当取代基为苯甲酰氧基时其对映选择性最好.

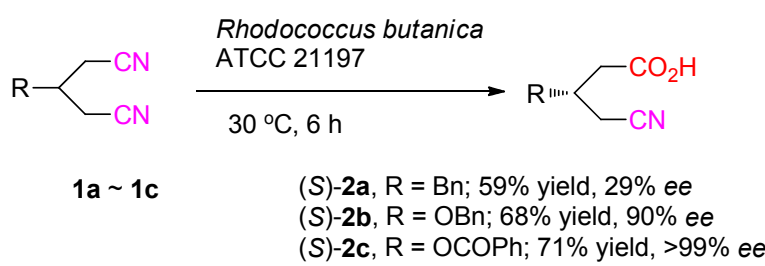

随后 Turner 课题组 ${ }^{[12]}$ 使用固定化整细胞催化剂 Rhodococcus SP 361, 将一系列羟基被保护的 3-差基戊 二腈 3 进行水解反应得到相应的单羧酸产物 4 (Eq. 2), 需要指出的是, 对于不含有苯环结构的底物 $\mathbf{3 c}$ 和 $3 \mathbf{d}$ 生 物转化后仍然可以得到手性产物, 说明此类酶与底物的 手性识别过程中底物上的苯环结构并不是必须存在的. 在反应过程中并没有观察到单氨基单酰胺产物的生成, 所以作者认为在这样一个含有腈水合酶和酰胺水解酶 的催化体系中, 底物 3 先经过具有 $S$ 选择性的腈水合酶 催化的去对称化反应生成单酰胺产物, 随后经不具有选 择性的酰胺水解酶快速转化生成最终产物 $\mathbf{4}$, 其中氧基 水合过程是决速步.

$\gamma$-氨基丁酸(简称 GABA)是脊椎动物中枢神经系统 中重要的抑制性神经递质, 其结构类似物往往具有药理

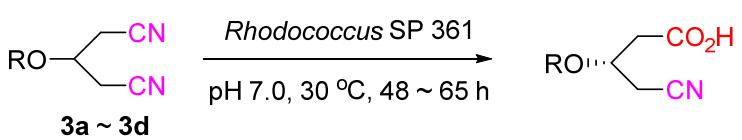

(S)-4a, R = Bn; 73\% yield, $83 \%$ ee

(S)-4b, R $=\mathrm{Bz} ; 25 \%$ yield, $84 \%$ ee

(S)-4c, R $=\mathrm{H} ; 52 \%$ yield, $22 \%$ ee

(S)-4d, R = MEM; $19 \%$ yield, $61 \%$ ee

活性, 例如药物普瑞巴林(Pregabalin)是一种亲脂性的 $\lambda-$ 氨基丁酸类似物, 可以用来治疗抑郁症、癫㾋以及社交 恐惧症等疾病; 药物巴氯芬(Baclofen, $\beta-4$-氯苯基 $-\gamma$-氨 基丁酸)是一种手性药物，曾作为肌松药用于临床，后 续研究发现其具有抗癫㾁、治疗三叉神经痛等临床作用, 研究表明 $R$-巴氯芬的活性远远高于 $S$-巴氯芬, 但由于光 学纯巴氯芬成本较高, 故市场上的药品是消旋体. 为了 实现手性巴氯芬尤其是 $R$-巴氯芬的合成, 2000 年前后, 王梅祥课题组 ${ }^{[13]}$ 开展了相关研究, 他们使用 Rhodococcus erythropolis AJ270 整细胞催化 3-取代戊二腈的水 解反应, 以中等到较高的对映选择性, 得到 $S$-3-取代-4氧基丁酸 6 (Scheme 4), 其中可以作为手性巴氯芬合成 前体的 $\mathbf{c}$ 其 $e e$ 可达 $63 \%$, 加入丙酮作为添加剂可以大 大提高产物的 $e e$ 值, 但是会导致反应效率的降低, 同时 当苯环上连有给电子基时, 反应具有较高的对映选择 性，以环已基作为取代基时，反应仍然具有很高的对映 选择性, 说明芳环对于酶和底物的手性识别并不起决定 作用. Rhodococcus erythropolis AJ270 含有腈水合酶和 酰胺水解酶, 且在反应过程中仍然没有观察到单氰基单 酰胺的生成, 所以该反应与之前所述 Rhodococcus SP 361 催化反应过程类似, 是有一个由具有 $\mathrm{S}$ 选择性的腈

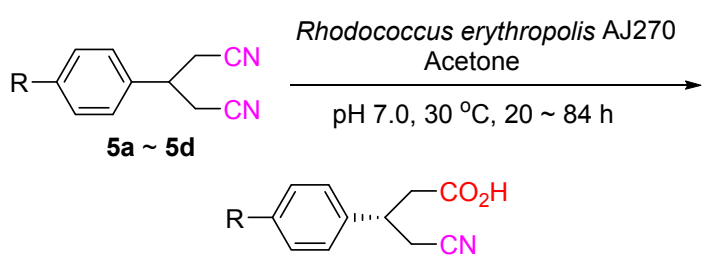

(S)-6a, $\mathrm{R}=\mathrm{CH}_{3} ; 25 \%$ yield, $95 \%$ ee

(S)-6b, R = F; $55 \%$ yield, $32 \%$ ee

(S)-6c, R $=\mathrm{Cl} ; 25 \%$ yield, $63 \%$ ee

(S)-6d, R = H; 67\% yield, $88 \%$ ee

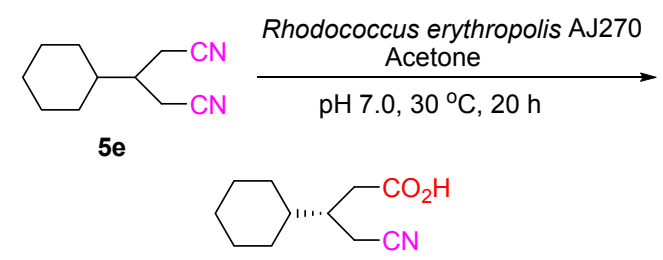

(S)-6e; $60 \%$ yield, $83 \%$ ee

图式 4 Rhodococcus erythropolis AJ270 催化龙二腈底物 5 的 去对称化生物转化反应

Scheme 4 Desymmetrization of prochiral glutaronitriles 5 by Rhodococcus erythropolis AJ270 
水合酶以及不具有选择性的酰胺水解酶实现的.

4-差基哌啶是多种天然产物和药物分子的基本骨 架, 为了合成手性 4-差基哌啶, 2002 年, Rutjes 课题组 ${ }^{[14]}$ 报道了相关研究工作(Scheme 5), 他们使用 Rhodococcus erythropolis NCIMB 11540 整细胞催化 3-苠氧基戊二腈 (7), 高效高产率地得到 $S$-3-芐氧基-4-氭基丁酸. 为了便 于分离和测量 $e e$ 值, 将其甲酯化得到相应产物 $\mathbf{8}$, 然后 通过简单化学转化获得 $S$-4-羟基-2-羰基哌啶(9). 该菌 株同时含有腈水解酶、腈水合酶和酰胺水解酶, 作者并 未指出此类底物的生物转化反应采取何种路径. 当使用 3-羟基戊二腈作为底物时, 反应的对映选择性大大降 低, 所得单氰基单羧酸酯 $e e$ 值为 $13 \%$, 说明底物羟基上 引入苄基后可以提高底物与酶的手性识别作用.

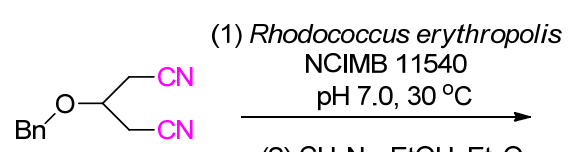

(2) $\mathrm{CH}_{2} \mathrm{~N}_{2}, \mathrm{EtOH}, \mathrm{Et}_{2} \mathrm{O}$

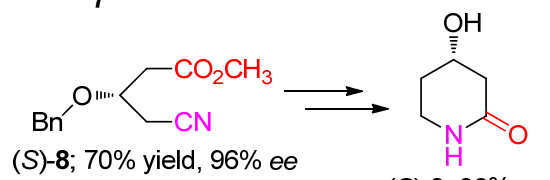

$(S)-9,96 \%$ ee

图式 5 化学-酶法合成手性 4-差基哌啶衍生物 9

Scheme 5 Chemoenzymatic approach to $S$-4-hydroxypiperidines 9

为了制备降胆固醇药物立普妥(Lipitor)的重要前体 $R$-3-羟基-4-氰基丁酸(11), 2002 年, Diversa 公司 ${ }^{[15]}$ 从其 基因库中笁选出一种具有 $R$ 选择性的腈水解酶, 可以将 3-差基戊二腈 10 去对称化转化为 $R-3$-羟基-4-氰基丁酸 (11), 其 $e e$ 值可达 $95 \%$, 但是在放大实验时产物的 $e e$ 值 降为 $88 \%$, 为了获得更加高效和高选择性的突变酶, 采 用基因饱和突变的方法, 通过大量笁选, 最终获得了可 以使产物 $e e$ 值达 $98 \%$ 的突变酶(Scheme 6). 同样为了合 成 $R$-3-差基-4-氰基丁酸(11), 2006 年, Bergeron 等 ${ }^{[16]}$ 报道 了从 Pseudomonas fluorescens 中提纯的腈水解酶 Nitrilase BD9750 催化 3-差基戊二腈(10)的水解反应, 在 $\mathrm{pH} 7.5$ 的条件下反应 $16 \mathrm{~h}$, 几乎以定量的产率生成光学 纯的单氰基羧酸 11 .

2009 年, Brady 课题组 ${ }^{[17]}$ 对 Rhodococcus rhodochrous ATCC BAA-870 催化 3-取代戊二腈 12 的去对称 化生物转化反应进行了研究(Eq. 3), 该反应需要加入甲 醇作为添加剂, 底物取代基为羟基时产物 $e e$ 值很低, 但 是羟基被苄基或苯甲酰基保护时可以高选择性地得到 单氰基羧酸产物 13, 此菌株含有腈水合酶和酰胺水解 酶, 作者认为 $13 \mathrm{~b} \sim 13 \mathrm{c}$ 产率较低可能是由于腈水合酶 不具有对映选择性, 而酰胺水解酶具有较高的对映选择 性, 底物 12 经过腈水合酶完全转化为外消旋的单氭基
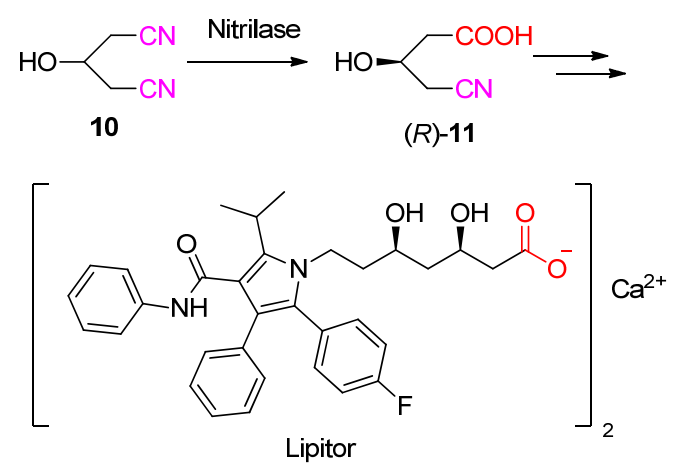

图式 6 化学-酶法合成 $R$-3-羟基-4-氧基丁酸 11

Scheme 6 Chemoenzymatic approach to $R$-4-cyano-3hydoxybutyric acid (11)

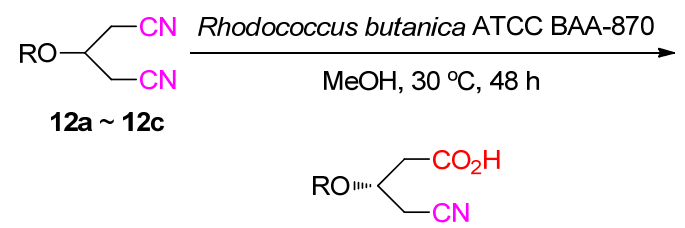

(S)-13a, $\mathrm{R}=\mathrm{H}$; $72 \%$ yield, $5 \%$ ee

(S)-13b, $\mathrm{R}=\mathrm{Bn} ; \mathbf{3 5 \%}$ yield, $92 \%$ ee

$(S)-13 c, R=B z ; 26 \%$ yield, $99 \%$ ee

单酰胺中间体，随后酰胺水解酶对其进行动力学拆分造 成 13 产率低于 $50 \%$.

2013 年, 许正宏课题组和朱敦明课题组 ${ }^{[18]}$ 共同报 道了生物催化 3-(4-氯苯基)-戌二腈去对称化生物转化 合成光学纯巴氯芬前体的研究工作，他们从实验室保藏 的含有腈水解酶的菌株中笁选出对这一底物具有较高 对映选择性的菌株 Gibberella intermedia WX12, 优化反 应条件后以 $90 \%$ 的产率得到 $S$-4-氰基-3-(4-氯苯基)-丁 酸, 其 $e e$ 值大于 $99 \%$. 随后, 朱敦明等 ${ }^{[19]}$ 进一步报道了 利用化学-酶法合成手性药物 $S$-Pregabalin 和 $R$-Baclofen 的研究工作(Scheme 7, 所示产率为气相产率), 他们篮 选了一系列具有腈水解酶的菌株, 并发现其中的 BjNIT6402 和 HsNIT 具有很高的 $S$ 选择性, 分别将底物

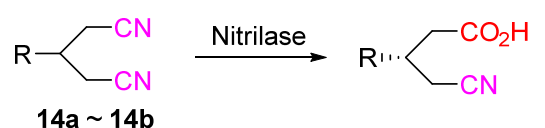

(S)-15a, R =|sobutyl; 100\% yield, 94\% ee (S)-15b, R = 4-Chlorophenyl; 99\% yield, $98 \%$ ee

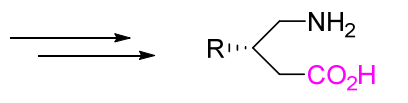

(S)-16a, S-Pregabalin (R)-16b, R-Baclofen

图式 7 化学-腈水解酶法合成 Pregabalin (16a)和 Baclofen (16b)

Scheme 7 Chemoenzymatic approach to $S$-pregabalin (16a) and $R$-baclofen (16b) 
14 与该两种酶反应, 都可以很高的转化率得到 $e e$ 值非 常高的羧酸产物 15 , 将其进行包括柯提斯重排反应在 内的简单化学转化即可得到手性药物 $S$-Pregabalin 和 $R$-Baclofen.

2013 年, Nojiri 课题组 ${ }^{[20]}$ 也报道了使用化学-酶法合 成这两种手性药物(Scheme 8), 与朱敦明等采取的途径 不同, 他们使用含有腈水合酶和酰胺水解酶的菌株 Comamonas sp. KNK 来实现 3-取代戊二酰胺 17 的去对 称化生物转化, 并以较高的产率和对映选择性得到 $R$ 构 型的单酰胺单羧酸产物 18 , 然后各自通过包括霍夫曼 重排反应在内的简单化学转化生成 $S$-Pregabalin 和 $R$-Baclofen.

\section{2 前手性丙二腈类底物的去对称化生物转化反 应}

丙二腈类底物的去对称化生物水解反应引起大家 的兴趣主要基于以下几点: 首先, 丙二腈类底物易于合 成，通常只需要丙二腈与卤代烷烃在碱的作用下反应即 可生成; 其次, 相比于戊二腈类底物, 丙二腈类底物的 氯基处于手性中心的 $\alpha$ 位, 反应位点与手性中心距离较 近更容易实现手性控制得到高光学纯产物; 最后, 生物 转化反应所生成的手性产物很容易通过简单化学反应 转化为 $\alpha$-氨基酸, 为各种天然和非天然氨基酸的合成提 供了有效途径. 因为当丙二腈及其水解产物的 $\alpha$ 位上有 氢原子时化合物容易发生消旋化, 所以该类反应底物主 要集中在 2,2-二取代丙二腈上.
1993 年, Ohta 小组 ${ }^{[21]}$ 首次报道了丙二腈类底物的去 对称化生物转化反应(Scheme 9), 他们使用之前提到的 Rhodococcus rhodochrous ATCC 21197(该菌株后来更名 为 Rhodococcus rhodochrous IFO 15564)整细胞在非常温 和的条件下水解 2-甲基-2-丁基丙二腈 19a 并以 94\%产 率、96\% ee 值得到 $R$ 构型的 2-酰胺基-2-甲基己酸 20a, 经过霍夫曼重排和浓盐酸水解反应后以 $78 \%$ 的产率生 成 $S$ 构型氨基酸 21a. 作者对生物转化过程进行了研究, 认为反应历程如 Scheme 9 所示, 底物 19 首先经过腈水 合酶的催化快速转化为二酰胺 22, 随后潜手性的二酰 胺 22 在具有 $R$ 选择性的酰胺水解酶作用下发生去对称 化转化生成 $R$ 构型的单酰胺单羧酸产物 20. 2004 年，该 课题组 ${ }^{[22]}$ 报道了后续工作，他们扩大底物范围，研究了 一系列 2,2-二取代丙二腈的去对称化生物转化反应(Eq. 4), 发现 Rhodococcus rhodochrous IFO 15564 在水解这 类底物时，反应的速率和对映选择性明显受到取代基位 阻及电性的影响. 其中, 取代基位阻越小, 反应速率越 高; 取代基 $\mathrm{R}$ 为烯丙基、苯基或苠基时产物羧酸 20 具 有很高 $e e$ 值 $(>95 \%)$. 当将丙二腈 $\alpha$ 位的甲基换为氟时, 反应的对映选择性大大降低，且产物组成较为复杂，不 再按照之前所述的催化途径进行转化. 甲基多巴 (S- $\alpha$-methyldopa) 可以治疗高血压, 并被用作孕妇高血 压的首选药物，作者展示该催化方法在有机合成方面的 应用(Eq. 5), 将去对称化生物转化反应所得到的产物 20h 进行甲酯化和霍夫曼重排后，以 $73 \%$ 的总产率得到 $e e$ 值为 $98 \%$ 的甲基多巴前体 $\mathbf{2 3}$.

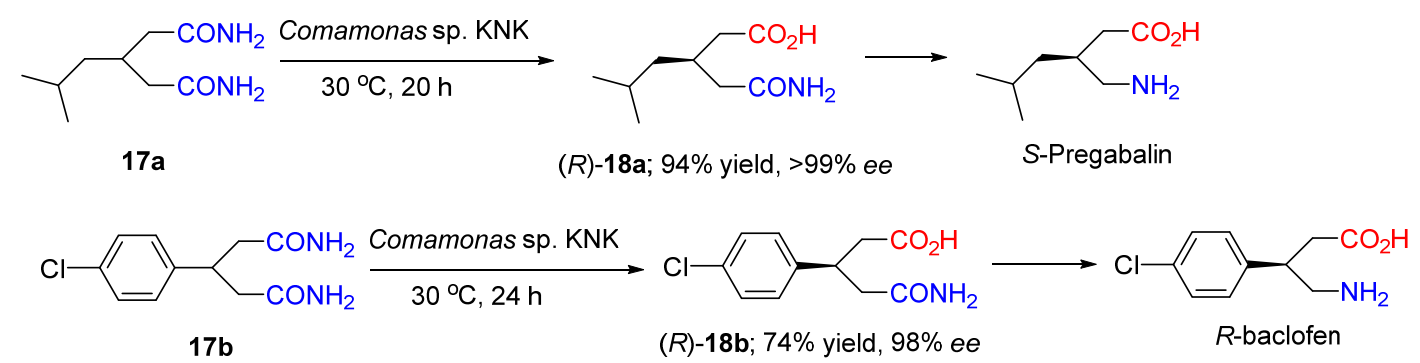

图式 8 化学-酰胺水解酶法合成 Pregabalin (16a)和 Baclofen (16b)

Scheme 8 Chemoenzymatic approach to $S$-pregabalin (16a) and $R$-baclofen (16b)

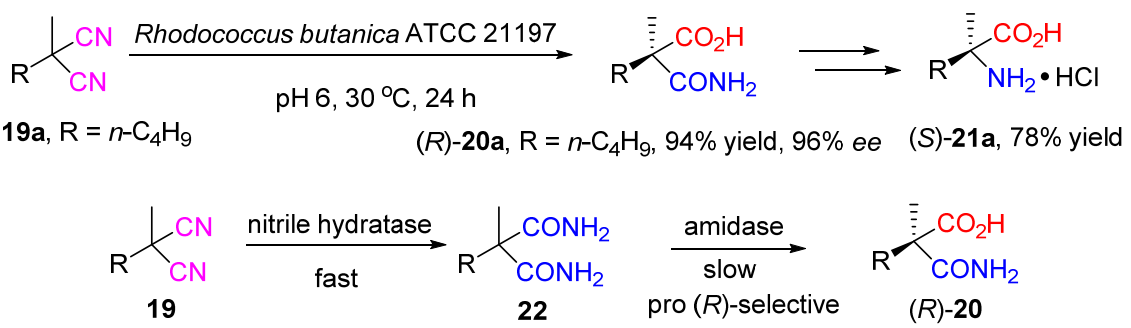

图式 9 Rhodococcus butanica ATCC 21197 催化丙二腈 19 的去对称化生物转化反应 Scheme 9 Desymmetrization of prochiral malonitriles 19 by Rhodococcus butanica ATCC 21197 


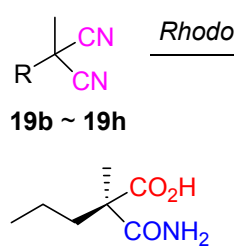

(R)-20b, $1 \mathrm{~d}$ $81 \%$ yield, $75 \%$ ee<smiles>CC(C)[C@](C)(C(N)=O)C(=O)O</smiles>

(R)-20e, $4 \mathrm{~d}$ $84 \%$ yield, $23 \%$ ee

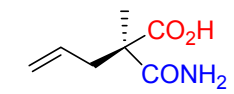

(R)-20c, $1 \mathrm{~d}$ $72 \%$ yield, $95 \%$ ee

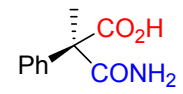

(R)-20f, $7 \mathrm{~d}$ $48 \%$ yield, $>99 \%$

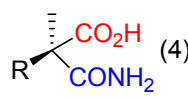

(R)-20b 20h

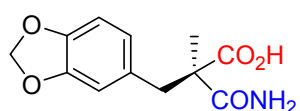

(R)-20h, $3 \mathrm{~d}$ $95 \%$ yield, $98 \%$

$$
\mathrm{Ph} \stackrel{\stackrel{\mathrm{F}}{\mathrm{K}} \mathrm{CONH}_{2} \mathrm{H}}{\mathrm{CO}_{2}}
$$

20i, 1

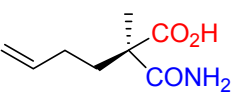

(R)-20d, 1 d $76 \%$ yield, $49 \%$ ee
(4) $6 \%$ yield, $13 \%$ ee

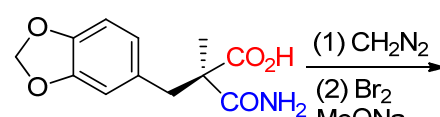

(R)-20h

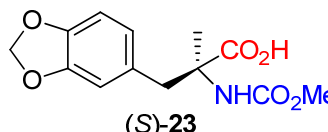

(S)-23
$73 \%$ yield, $98 \%$ ee

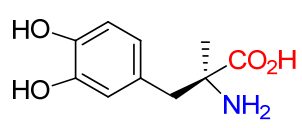

24, (S)- $\alpha$-methyldopa

2003 年, 李祖义课题组 ${ }^{[23]}$ 连续发表论文, 他们使用 含有腈水合酶和酰胺水解酶的菌株 Rhodococcus sp. CGMCC 0497, 分别催化 2-甲基-2-芐基丙二腈(25)和 2甲基-2-芐基丙二酰胺(27)的水解反应, 均以较高产率得 到高光学纯的单酰胺单羧酸产物 26 (Scheme 10), 当反 应在 $20{ }^{\circ} \mathrm{C}$ 进行时其效率和选择性都要优于 $30{ }^{\circ} \mathrm{C}$ 时. 当以丙二腈类化合物 $\mathbf{2 5}$ 作为底物时, 反应速率较低, 需 要 $7 \mathrm{~d}$ 才能完成反应, 且当苯环上连有给电子取代基 (25b 25c) 或者苯环邻位含有取代基 (25h) 时, 产率较 低; 当以丙二酰胺类化合物 $\mathbf{2 7}$ 为底物时, 反应速率大大 提高, 仅需要 $1 \mathrm{~d}$ 就能以 $90 \%$ 以上的产率得到相应产物 26. 作者对反应机制也进行了考察(Scheme 11), 以 2-甲 基-2-芐基丙二腈 (25a)为底物在 $30{ }^{\circ} \mathrm{C}$ 下进行生物转化 反应, 反应 $66 \mathrm{~h}$ 后, 可以得到单氧基酰胺 $S-28 \mathrm{a}$ (产率 $56 \%, e e$ 值 $93 \%$ )、单氧基羧酸 $R-29 \mathbf{a}$ (产率 $33 \%, e e$ 值 $52 \%$ ) 和二酰胺 $27 \mathbf{a}($ 产率 $8 \%$ ) 的混合物; 反应 $112 \mathrm{~h}$ 后, 可以得 到单氰基羊酸 29a(产率 44\%, ee 值 1\%) 和单酰胺单羧酸 26a(产率 52\%, ee 值 95\%) 的混合物, 可见在 $30{ }^{\circ} \mathrm{C}$ 下进 行反应其历程较为复杂且有大量接近于外消旋的单氰 基羧酸 29 生成, 降低温度可以大大抑制 29 的生成. 结 合产物的绝对构型可推测 Rhodococcus sp. CGMCC 0497 中的腈水合酶具有较低的反应速率和较低的 $S$-选 择性, 酰胺水解酶具有较高的反应速率和较高的 $R$-选

择性.

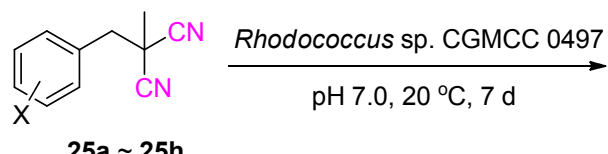

$25 a \sim 25 h$

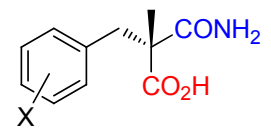

(R)-26a, $\mathrm{X}=\mathrm{H}$; $96 \%$ yield, $>99 \%$ ee

(R)-26b, $\mathrm{X}=p-\mathrm{CH}_{3} ; 58 \%$ yield, $>99 \%$ ee

(R)-26c, $\mathrm{X}=p-\mathrm{OCH}_{3} ; 58 \%$ yield, $>99 \%$ ee

(R)-26d, $\mathrm{X}=p$-F; $80 \%$ yield, $>99 \%$ ee

(R)-26e, $\mathrm{X}=p$-Cl; $83 \%$ yield, $>99 \%$ ee

(R)-26f, $\mathrm{X}=p$-Br; $81 \%$ yield, $>99 \%$ ee

(R)-26g, $\mathrm{X}=m$-Cl; $85 \%$ yield, $98 \%$ ee

(R)-26h, $\mathrm{X}=o-\mathrm{Cl}$; $65 \%$ yield, $99 \%$ ee

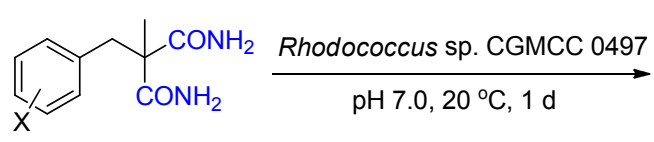

$27 a \sim 27 h$

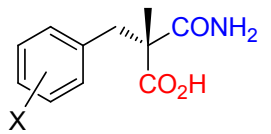

(R)-26a, $\mathrm{X}=\mathrm{H} ; 94 \%$ yield, $97 \%$ ee

$(R)-26 \mathbf{b}, \mathrm{X}=p-\mathrm{CH}_{3} ; 98 \%$ yield, $>99 \%$ ee

(R)-26c, $\mathrm{X}=p-\mathrm{OCH}_{3} ; 93 \%$ yield, $>99 \%$ ee

(R)-26d, $\mathrm{X}=p-\mathrm{F} ; 95 \%$ yield, $>99 \%$ ee

$(R)-\mathbf{2 6 e}, \mathrm{X}=p$-Cl; $95 \%$ yield, $>99 \%$ ee

(R)-26f, $\mathrm{X}=p$-Br; $94 \%$ yield, $>99 \%$ ee

$(R)-\mathbf{2 6 g}, \mathrm{X}=m-\mathrm{Cl} ; 91 \%$ yield, $96 \%$ ee

$(R)-\mathbf{2 6 h}, \mathrm{X}=\mathrm{o}-\mathrm{Cl} ; 92 \%$ yield, $97 \%$ ee

图式 10 Rhodococcus sp. CGMCC 0497 催化丙二腈 $\mathbf{2 5}$ 和丙二 酰胺 27 的去对称化生物转化反应

Scheme 10 Desymmetrization of prochiral malonitriles 25 and malonamides 27 by Rhodococcus sp. CGMCC 0497
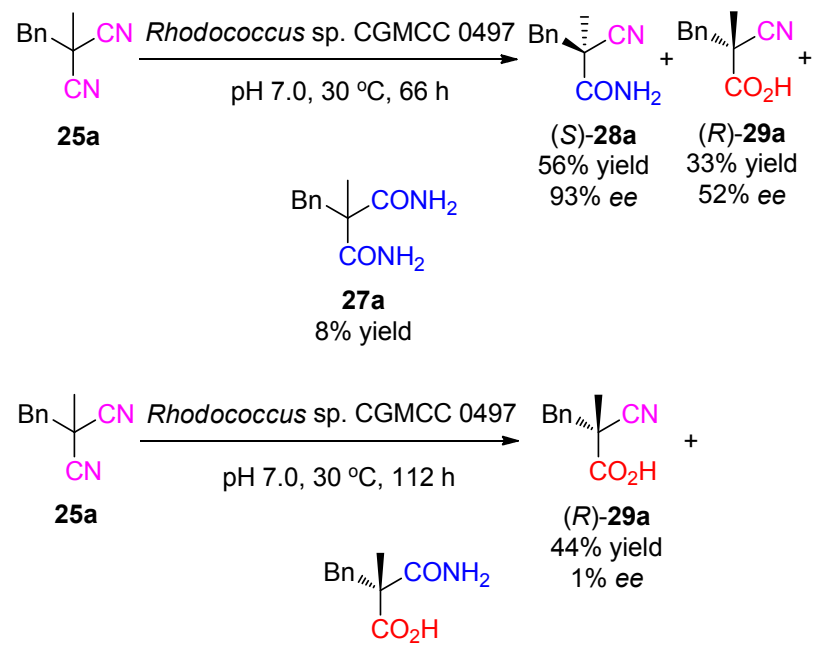

(R)-26a

$52 \%$ yield

$95 \%$ ee

图式 11 Rhodococcus sp. CGMCC 0497 催化机制的探究

Scheme 11 Research for mechanism of the desymmetrizations by Rhodococcus sp. CGMCC 0497 
2006 年, Rutjes 小组 ${ }^{[24]}$ 对二取代丙二腈类底物 30 的 去对称化生物转化反应进行了初步研究(Eq. 6), 他们使 用 Rhodococcus erythropolis NCIMB 11540 整细胞催化 得到了一系列单氰基单酰胺产物 31, 其中取代基为正 戊基时可以较高的产率得到 ee 值高达 $98 \%$ 的产物 $31 \mathrm{c}$ (作者并未给出产物绝对构型), 说明该菌株的腈水 合酶对于特定的底物具有较高对映选择性.

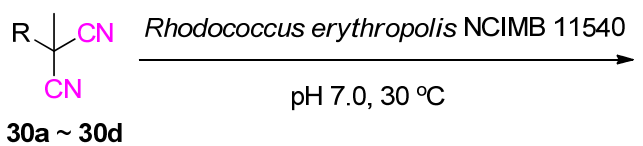

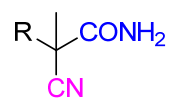

31a, $\mathrm{R}=\mathrm{Ph} ; 90 \%$ yield, $15 \%$ ee 31b, $\mathrm{R}=n-\operatorname{Pr} ; 65 \%$ yield, $58 \%$ ee 31c, $\mathrm{R}=n$-heptyl; $85 \%$ yield, $98 \%$ ee 31d, $R=B n ; 94 \%$ yield, $23 \%$ ee

2011 年, 王梅祥课题组使用含有腈水合酶和酰胺 水解酶的菌株 Rhodococcus erythropolis AJ270 实现了二 烷基取代丙二酰胺 32 的去对称化生物水解反应 ${ }^{[25]}$, 以 较高产率得到高光学纯的单酰胺单羧酸产物, 为了便于 测定 $e e$ 值, 将其酯化为羧酸酯产物 33(Eq. 7), 降低生物 转化反应的温度可以提高对映选择性, 但是会降低反应 速度和产率. 当底物上取代基含有双键或苯环时, 产物 $e e$ 值较高, 当取代基为位阻较大的基团时, 反应效率和 对映选择性都较低. 随后, 他们发现把取代基上的甲基 换为氨基后可以大大提高反应的效率和对映选择性 ${ }^{[26]}$ (Eq. 8), 且不论取代基上是否含有双键或苯环, 都可以 在 $1 \mathrm{~h}$ 内完成反应得到 $e e$ 值大于 $95 \%$ 的产物(只有 $\mathbf{3 4 g}$ 例外). 分析比较生物转化产物 $\mathbf{3 3}$ 和 $\mathbf{3 5}$ 的构型可以发 现, 虽然产物都是 $R$ 构型, 但是显然酰胺水解酶与这两 种底物的作用机制不同, 当底物不含有氨基时, 底物取 代基的立体效应和电子效应共同决定了酶与其手性识 别作用; 当底物上含有氨基时, 氨基与酶有更强的手性 识别作用.

\section{3 内消旋环状二腈类底物的去对称化生物转化} 反应

相比于前手性二腈, 关于内消旋环状二腈的去对称 化生物转化反应的报道较少, 这一方面是由于高效合成 内消旋环状二腈底物的化学方法不多, 特别是含有多官 能团的底物其合成有时较为困难, 另一方面是由于其手 性产物在合成天然产物或药物上的应用有待开发.

1998 年, Sugai 和 Ohta 等 ${ }^{[27]}$ 使用 Rhodococcus rhodochrous IFO 15564 整细胞催化内消旋的六元脂环二腈 及二酰胺的生物转化反应, 可以得到相应的水解产物,
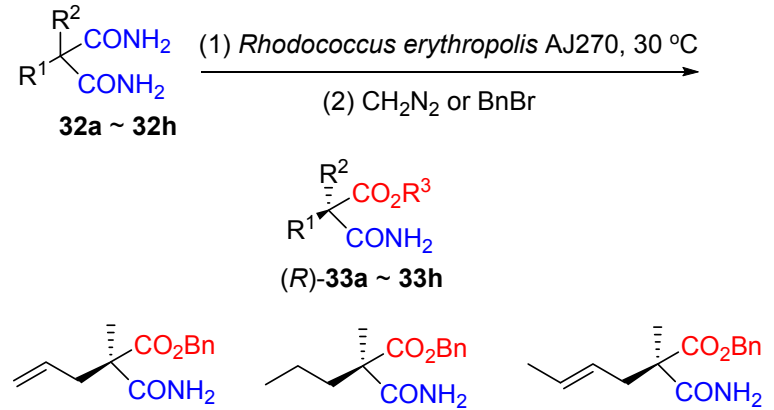

(R)-33a, $0.5 \mathrm{~h}$

(R)-33b, $5 \mathrm{~h}$

$(R)-33 \mathrm{c}, 1.3 \mathrm{~h}$

$85 \%$ yield, $91 \%$ ee $80 \%$ yield, $72 \%$ ee

$87 \%$ yield, $98 \%$ ee

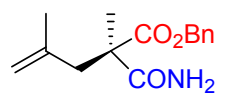

(R)-33d, $0.7 \mathrm{~h}$
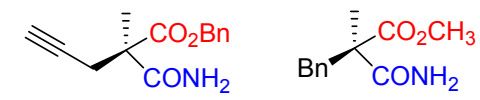

(R)-33e, $25 \mathrm{~h}$

(R)-33f, $8 \mathrm{~h}$

$85 \%$ yield, $43 \%$ ee $73 \%$ yield, $44 \%$ ee $99 \%$ yield, $>99.5 \%$ ee

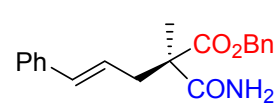

(R)-33g, $115 \mathrm{~h}$ $84 \%$ yield, $>99.5 \%$ ee

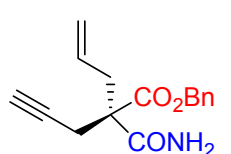

$(R)-33 \mathrm{~h}, 31.5 \mathrm{~h}$

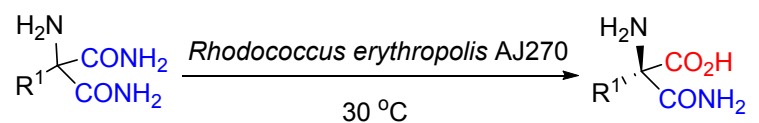

$34 a \sim 34 h$<smiles>C=CC[C@](N)(C(N)=O)C(=O)O</smiles>

(R)-35a, $40 \mathrm{~min}$ $87 \%$ yield, $97 \%$ ee<smiles>C=CCC[C@](N)(C(N)=O)C(=O)O</smiles>

(R)-35d, $25 \mathrm{~min}$

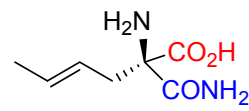

(R)-35b, $50 \mathrm{~min}$ $93 \%$ yield, $97 \%$ ee

$$
\approx \stackrel{\mathrm{H}_{2} \mathrm{~N}}{\mathrm{~L}_{\mathrm{CO}} \mathrm{H}}
$$

(R)-35e, $25 \mathrm{~min}$ $80 \%$ yield, $97 \%$ ee
(R)-35a $\sim 35 \mathrm{~h}$<smiles>C=C(C)C[C@](N)(C(N)=O)C(=O)O</smiles>

(R)-35c, $40 \mathrm{~min}$ $98 \%$ yield, $99 \%$ ee

$$
\mathrm{Bn}^{\prime \prime}{ }_{\mathrm{CONH}_{2}}^{\mathrm{H}_{2} \mathrm{~N}}
$$

(R)-35f, $60 \mathrm{~min}$ $83 \%$ yield, $>99.5 \%$ ee

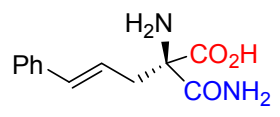

(R)-35g, $330 \mathrm{~min}$ $74 \%$ yield, $94 \%$ ee

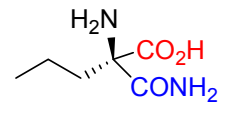

(R)-35h, $35 \mathrm{~min}$ $84 \%$ yield, $98 \%$ ee
底物的骨架结构和是否含有双键对反应的速率和立体 选择性有较大影响, 当底物结构上含有桥环 $\mathbf{3 6}$ 时, 可以 $76 \%$ 产率和 $74 \%$ ee 值得到单氧基羧酸产物 37 ; 底物上 不含有桥环时(底物 38, 42), 可以生成高光学纯的单酰 胺羧酸和二羧酸产物, 直接以二酰胺 41 作为底物, 可以 提高产物 39 的 $e e$ 值. 对反应的过程进行详细研究后作 者给出了反应历程, 他们认为二腈底物 $\mathbf{3 8}$ 在不具有对 映选择性的腈水合酶作用下转化为二酰胺 41 , 然后在 酰胺水解酶的作用下先后生成单羧酸和二羧酸产物, 反 应的对映选择性主要来源于酰胺水解酶(Scheme 12). 


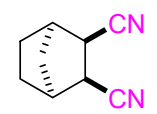

36

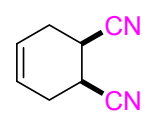

38

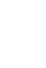
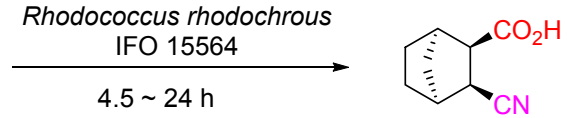

$37,76 \%$ yield, $74 \%$ ee
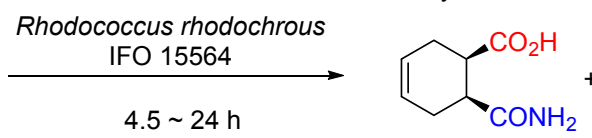

39, $39 \%$ yield, $83 \%$ ee

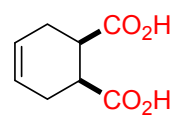

40, $33 \%$ yield

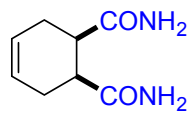

Rhodococcus rhodochrous
IFO 15564

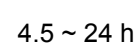

41

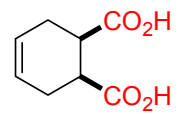

40, 39\% yield

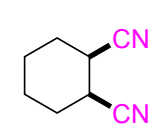

$\underset{\text { IFO } 15564}{\stackrel{\begin{array}{c}\text { Rhodococcus rhodochrous } \\ \text { IFO }\end{array}}{4.5 \sim 24 \mathrm{~h}}}$

42

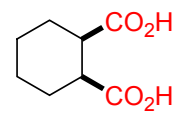

$44,24 \%$ yield

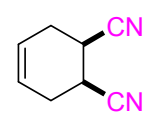

38
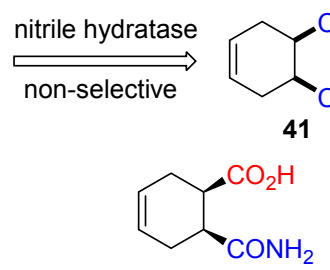

$(1 R, 6 S)-39$

图式 12 Rhodococcus rhodochrous IFO 15564 催化内消旋的 六元环状二腈的水解

Scheme 12 Desymmetrization of meso cyclic dinitriles by Rhodococcus rhodochrous IFO 15564

2012 年, 王梅祥课题组 ${ }^{[28]}$ 报道了 Rhodococcus erythropolis AJ270 整细胞催化内消旋氮杂环二酰胺 $\mathbf{4 2}$ 的去 对称化生物转化反应(Eq. 9), 反应以非常高的对映选择 性生成单羒酸产物 43 , 随着氮上取代基位阻的增加, 反 应时间延长且产率降低. 当底物环上引入双键(42e) 时, 其效率和对映选择性仍然很高, 但是酶催化七元环底物 (42g) 表现出了较低的对映选择性. 值得一提的是, 酶催 化 2,5-二酰胺基四氢吡咯 42a 的去对称化反应效率非常 高, 可以 $94 \%$ 的产率一锅得到 $19 \mathrm{~g}$ 对映体纯产物 2-羧基 -5-酰胺基四氢吡咯(43h). 随后, 该课题 组 ${ }^{[29]}$ 又以相 似的方法研究内消旋 1,3-环戊二腈和 1,3-环戊二酰胺的 去对称化生物转化反应, 与之前所述内消旋氮杂环二酰 胺底物 42 的生物转化反应相比, 底物上两个取代基的
位阻造成反应速率较低. 研究发现腈水合酶可以低的 $1 R$-对映选择性高效催化 1,3 -环戊二腈及衍生物的单氧 基水和反应，而酰胺水解酶以高的 $1 S$-对映选择性催化 1,3-环戊二酰胺 44 的单酰胺水解反应，并以最高可达 94\%的产率生成高光学纯度的单酰胺单羧酸产物 45 (Eq. 10), 反应速率受到底物上取代基位阻效应的较大 影响，尤其当大位阻取代基与酰胺基处于顺式时反应速 率很低，而底物上取代基类型和位置都不影响其非常高 的对映选择性.

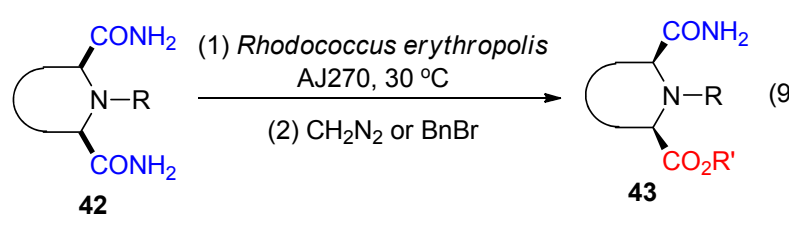<smiles>CC(=O)C1CCC(C(N)=O)N1</smiles>

43a, $1 \mathrm{~h}$ $96 \%$ yield, $96 \%$ ee<smiles>COC(=O)[C@@H]1CC[C@@H](C(N)=O)N1Cc1ccccc1</smiles>

43d, $96 \mathrm{~h}$<smiles>COC1CCC(C(N)=O)N1C</smiles>

43b, $60 \mathrm{~h}$ $80 \%$ yield, $60 \%$ ee

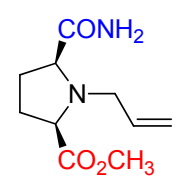

43c, $96 \mathrm{~h}$ $60 \%$ yield, $95 \%$ ee
$15 \%$ yield, $>99.5 \%$ ee $\quad 96 \%$ yield, $>99.5 \%$ ee $90 \%$ yield, $>99.5 \%$ ee<smiles>NC(=O)C1CCCCC(C(=O)OCc2ccccc2)N1</smiles>

43g, $1 \mathrm{~h}$<smiles>NC(=O)[C@@H]1C=C[C@@H](C(=O)OBr)N1</smiles>

$$
\mathrm{CO}_{2} \mathrm{Bn}
$$

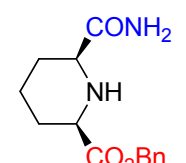

$43 \mathrm{f}, 1 \mathrm{~h}$
同时王梅祥课题组还展示了生物转化方法在合成 具有潜在药物活性化合物上的应用 ${ }^{[28,29]}$ (Scheme 13), 他 们以去对称化生物转化反应得到的手性产物 $43 \mathrm{~h}$ 为原 料, 通过几步简单的官能团转化反应，以较高的总产率 合成了氮杂核苷类似物 49 以及苯并-1,4-二氮杂草酮化 合物 51; 还以具有多个手性中心的产物 45a 为原料, 构 筑含有多官能团的环戊烷并 $\delta$-内酰胺化合物 $\mathbf{5 2}$.

\section{4 其它类型前手性底物的去对称化生物转化反 应}

除了碳原子可以作为手性中心外, 磷原子和硫原子 也可以作为手性中心，而基于磷或硫手性中心的去对称 化生物转化反应也有人报道. 2007 年, Rutjes 课题组 ${ }^{[30]}$ 报道了使用几种商品化的腈水解酶, 进行两类底物二乙 腈基苯基氧膦(53)和二乙腈基亚砜(56)的去对称化生物 转化反应(Scheme 14), 反应产物以单氰基酰胺和单氭 基羧酸为主, 其中对于底物 53, 腈水解酶 Nitrilase 106 


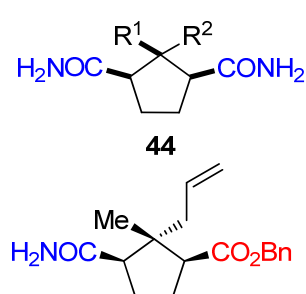

45a, $42 \mathrm{~h}$ $93 \%$ yield, $>99.5 \%$ ee

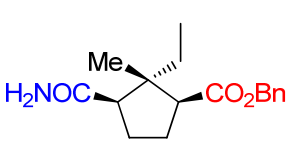

45e, $30 \mathrm{~h}$

$90 \%$ yield, $>99.5 \%$ ee
(1) Rhodococcus erythropolis AJ270, $30^{\circ} \mathrm{C}$

(2) $\mathrm{BnBr}$<smiles>C/C=C1\C2CCC(C(=O)OCc3ccccc3)C1(C)CC2N</smiles>

45b, $46 \mathrm{~h}$ $91 \%$ yield, $>99.5 \%$ ee<smiles>C[C@@]1(CCO)[C@H](C(N)=O)CC[C@H]1C(=O)OBr</smiles>

45f, $168 \mathrm{~h}$ $0 \%$ yield<smiles>[R]C1([R])C(C(N)=O)CCCC1C(N)=O</smiles>

45<smiles>C=CC12CC(CCC1C(=O)OCc1ccccc1)C(C(N)=O)C2</smiles>

$45 \mathrm{c}, 38 \mathrm{~h}$ $92 \%$ yield, $>99.5 \%$ ee<smiles>C=CC[C@]1(C)[C@H](C(N)=O)CC[C@H]1C(=O)OCc1ccccc1</smiles>

45g, $168 \mathrm{~h}$ $55 \%$ yield, $>99.5 \%$ ee

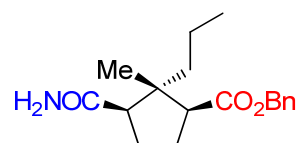

45d, $37 \mathrm{~h}$ $92 \%$ yield, $>99.5 \%$ ee<smiles>CC(=O)OC1CCC(C(N)=O)C1(C)Cc1ccccc1</smiles>

45h, $168 \mathrm{~h}$ $39 \%$ yield, $>99.5 \%$ ee

$$
\text { }
$$

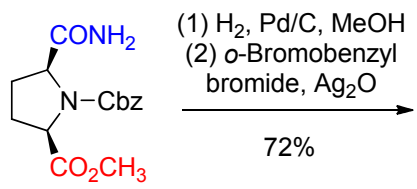

46<smiles>CC(=O)OC1CCC(C#N)N1C(=O)O</smiles>

47

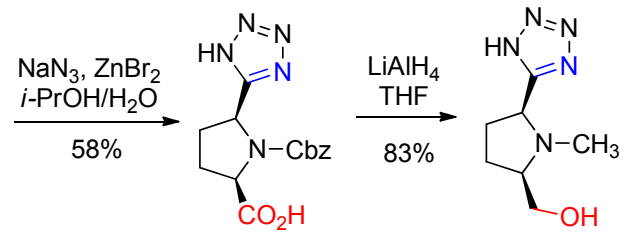

48

49

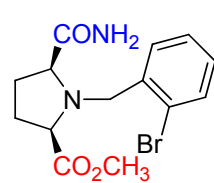

50<smiles>O=C1Nc2ccccc2CN2C(CO)CCC12</smiles>

51<smiles>C=CC[C@@]1(C)CC(C(=O)OCc2ccccc2)CC1C(N)=O</smiles>

(1) $\mathrm{O}_{3}$

(2) $\mathrm{Me}_{2} \mathrm{~S}$

$\stackrel{\text { (3) } \mathrm{Et}_{3} \mathrm{SiH}, \mathrm{BF}_{3} \cdot \mathrm{OEt}_{2}}{\longrightarrow}$

(1) $\mathrm{NaBH}_{4}, \mathrm{LiCl}$

(2) Cul, DMGC

$80 \%$

$76 \%$

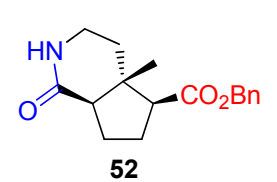

图式 13 去对称化生物转化手性产物在有机合成中的应用

Scheme 13 Synthetic applications of desymmetrization

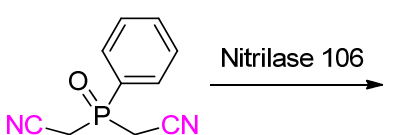

53

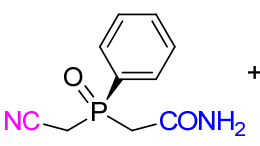

(S)-54 $11 \%$ yield, $>99 \%$ ee<smiles>C[SH](=O)(CC#N)CC(N)=O</smiles>

(S)-57

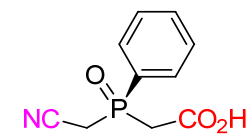

(S) -55

$51 \%$ yield, $70 \%$ ee<smiles>C[SH](=O)(CC#N)CC(=O)O</smiles>

(R)-58 $20 \%$ yield, $77 \%$ ee

图式 14 以杂原子为手性中心底物的去对称化生物转化反应

Scheme 14 Desymmetrization of prochiral dinitriles by nitrilase

表现出最好的对映选择性, 生成对映体纯的单氰基酰胺 产物 54; 而对于底物 56, 腈水解酶 Nitrilase 104 表现出 最好的对映选择性, 生成对映体纯的单氰基酰胺产物 57. 作者认为所生成的羧酸产物并不是由酰胺产物进一 步转化生成的, 而是同时由㲵基转化而来. 值得一提的 是, 腈水解酶纯酶催化腈类底物反应后生成酰胺这一反
常现象并非个例, Sheldon 课题组 ${ }^{[31]}$ 在 2006 年就报道了 重组腈水解酶具有腈水合酶活性并针对这一现象给出 了机理上的解释.

\section{5 结论与展望}

经过二十几年的发展，前手性或内消旋腈类化合物 
的去对称化生物转化反应已经成为合成手性酰胺和羧 酸的重要方法, 和其它方法相比具有明显优势, 比如相 比于动力学拆分的方法去对称化反应理论产率可达 $100 \%$; 底物腈化物可以通过多种成熟而又简单的方法 制备; 反应条件温和且选择性高不会破坏底物上的敏感 官能团, 而这些优势使得去对称化生物转化方法越来越 成为制备其它化学方法不易制得的多官能化手性酰胺 和羧酸的优选方法. 可以预见: 随着新型含有高效高对 映选择性腈的转化酶的菌株的不断发现, 随着生物技术 尤其是基因突变和重组技术的不断发展, 将为腈的去对 称化生物转化领域的蓬勃发展注入新的活力; 而对新型 前手性或内消旋二腈底物尤其是内消旋环状二腈底物 去对称化生物转化反应的不断探索将进一步拓宽这一 领域; 在化学生物学家的共同努力下, 酶催化这类反应 的作用机制尤其是催化过程中分子层面上的手性识别 机制将不断被探索认识, 并将进一步指导底物分子理性 设计; 而以去对称化生物转化方法得到的多官能化手性 酰胺羧酸将被更多地用来合成天然产物和具有生物活 性的化合物.

\section{References}

[1] Faber, K. Biotransformations in Organic Chemistry, 6th ed., Springer, Berlin, 2011

[2] Rappoport, Z.; Patai, S. The Chemistry of Functional Groups, The Chemistry of the Cyano Group, Wiley, London, 1970.

[3] (a) Evgred, D.; Harnett, S. Cyanide Compounds in Biology (Ciba Foundation Symposium 140), Wiley, Chichester, 1998.

(b) Jallageas, J.-C.; Arnaud, A.; Galzy, P. Adv. Biochem. Eng. 1980, 12,1 .

(c) Legras, J.-L.; Chuzel, G.; Arnaud, A.; Galzy, P. World J. Microbiol. Biotechnol. 1990, 6, 83.

[4] (a) Harper, D. B. Biochem. Soc. Trans. 1976, 4, 502.

(b) Harper, D. B. Biochem. J. 1977, 165, 309.

(c) Kobayashi, M.; Shimizu, S. FEBS Microbiol Lett. 1994, 120, 217.

[5] (a) Asano, Y.; Tani, Y.; Yamada, H. Agric. Biol. Chem. 1980, 44, 2251.

(b) Asano, Y.; Tachibana, Y.; Tani, Y.; Yamada, H. Agric. Biol. Chem. 1982, 46, 1175.

[6] (a) Brenner, C. Curr. Opin. Struct. Biol. 2002, 12, 775.

(b) Liu, Z. -Q.; Dong, L. -Z.; Cheng, F.; Xue, Y. -P.; Wang, Y. -S.; Ding, J. -N.; Zheng, Y. -G.; Shen, Y. -C. J. Agric. Food Chem. 2011, 59,11560 .

[7] (a) Mascharak, P. K. Coord. Chem. Rev. 2002, 225, 201.

(b) Song, L. Y.; Wang, M. Z.; Shi, J. J.; Xue, Z. Q.; Wang, M. -X.; Qian, S. J. Biochem. Biophy. Res. Commun. 2007, 362, 319.

[8] (a) Fournand, D.; Arnaud, A. J. Appl. Microbiol. 2001, 91, 381. (b) Ohtaki, A.; Murata, K.; Sato, Y.; Noguchi, K.; Miyatake, H.; Dohmae, N.; Yamada, K.; Yohda, M.; Odaka, M. Biochim. Biophys. Acta 2010, 1804, 184.

[9] (a) Sugai, T.; Yamazaki, T.; Yokoyama, M.; Ohta, H. Biosci. Biotechnol. Biochem. 1997, 61, 1419.

(b) Martínková, L.; Křen, V. Biocatal. Biotrans. 2002, 20, 73.

(c) Banerjee, A.; Sharma, R. Banerjee, U. C. Appl. Microbiol. Bio- technol. 2002, 60, 33.

(d) Wang, M.-X. Top. Catal. 2005, 35, 117.

(e) Martínková, L.; Uhnáková, B.; Pátek, M.; Nešvera, J.; Křen, V. Rhodococcus. Environ. Int. 2009, 35, 162.

(f) Wang, M.-X. Chimia 2009, 63, 331.

(g) Prasad, S.; Bhalla, T. C. Biotechnol. Adv. 2010, 28, 725;

(h) Velankar, H.; Clarke, K. G.; du Preez, R.; Cowan, D. A.; Burton, S. G. Trends Biotechnol. 2010, 28, 561.

(i) Wang, M.-X. Top. Organomet. Chem. 2011, 36, 105.

(j) Ramteke, P. W.; Maurice, N. G.; Joseph, B.; Wadher, B. J. Biotechnol. Appl. Biochem. 2013, 60, 459.

(k) Wang, M.-X. Acc. Chem. Res. 2015, 48, 602.

[10] (a) Garcia-Urdiales, E.; Alfonso, I.; Gotor, V. Chem. Rev. 2005, $105,313$.

(b) Palomo, J. M.; Cabrera, Z. Curr. Org. Synth. 2012, 9, 791.

[11] Kakya, H.; Sakai, N.; Yokoyama, M.; Sugai, T.; Ohta, H. Chem. Lett. 1991, 1823.

[12] (a) Crosby, J. A.; Parratt, J. S.; Turner, N. J. Tetrahedron: Asymmetry 1992, 3, 1547.

(b) Beard, T.; Cohen, M. A.; Parratt, J. S.; Turner, N. J.; Crosby, J.; Moilliet, N. J. Tetrahedron: Asymmetry 1993, 4, 1085.

[13] (a) Wang, M.-X.; Liu, C.-S.; Li, J.-S. Meth-Cohn, O. Tetrahedron Lett. 2000, 41, 8549 .

(b) Wang, M.-X.; Liu, C.-S.; Li, J.-S. Tetrahedron: Asymmetry 2002, $12,3367$.

[14] Vink, M. K. S.; Schortinghuis, C. A.; Luten, J.; van Maarseveen, J. H.; Schoemaker, H. E.; Hiemstra, H.; Rutjes, F. P. J. T. J. Org. Chem. 2002, 67, 7869 .

[15] (a) Santis, D. G.; Zhu, Z. L.; Greenberg, W. A.; Wong, K.; Chaplin, J.; Hanson, S. R.; Farwell, B.; Nicholson, L. W.; Rand, C. L.; Weiner, D. P.; Robertson, D. E.; Burk, M. J. J. Am. Chem. Soc. 2002, 124, 9024.

(b) Santis, D. G.; Wong, K.; Farwell, B.; Chatman, K.; Zhu, Z. L.; Tomlinson, G.; Huang, H.; Tan, X.; Bibbs, L.; Chen, P.; Kretz, K.; Burk, M. J. J. Am. Chem. Soc. 2003, 125, 11476.

[16] Bergeron, S.; Chaplin, D. A.; Edwards, J. H.; Ellis, B. S. W.; Hill, C. L.; Karen, H.-T.; Knight, J. R.; Mahoney, T.; Osborne, A. P.; Ruecroft, G. Org. Process Res. Dev. 2006, 10, 661.

[17] Kinfe, H. H.; Chhiba, V.; Frederick, J.; Bode, M. L.; Mathiba, K.; Steenkamp, P. A.; Brady, D. J. Mol. Catal. B: Enzym. 2009, 59. 231.

[18] Xu, M. Z.; Ren, J.; Gong, J. S.; Dong, W. Y.; Wu, Q. Q.; Xu, Z. H.; Zhu, D. M. Chin. J. Biotechnol. 2013, 29, 31 (in Chinese). (许美珍, 任杰, 龚劲松, 董文玥, 吴洽庆, 许正宏, 朱敦明, 生 物工程学报, 2013, 29, 31.)

[19] Duan, Y. T.; Yao, P. Y.; Ren, J.; Han, C.; Li, Q.; Yuan, J.; Feng, J. H.; Wu, Q. Q.; Zhu, D. M. Sci. Chin. Chem. 2014, 57, 1164.

[20] Nojiri, M.; Uekita, K.; Ohnuki, M.; Taoka, N.; Yasohara, Y. J. Appl. Microbiol. 2013, 115, 1127.

[21] Yokoyama, M.; Sugai, T.; Ohta, H. Tetrahedron: Asymmetry 1993, $4,1081$.

[22] Yokoyama, M.; Kashiwagi, M.; Iwasaki, M.; Fuhshuku, K.; Ohta, H.; Sugai, T. Tetrahedron: Asymmetry 2004, 15, 2817.

[23] (a) Wu, Z. -L.; Li, Z. -Y. Chem. Commun. 2003, 386. (b) Wu, Z. -L.; Li, Z. -Y. J. Org. Chem. 2003, 68, 2479. (c) Wu, Z. -L.; Li, Z. -Y. Tetrahedron: Asymmetry 2003, 14, 2133.

[24] Vink, M. K. S.; Wijtmans, R.; Reisinger, C.; Berg, R. J. F.; Schortinghuis, C. A.; Schwab, H.; Schoemaker, H. E.; Rutjes, F. P. J. T. Biotechnol. J. 2006, 1, 569.

[25] Zhang, L.-B.; Wang, D.-X.; Wang, M.-X. Tetrahedron 2011, 67, 5604.

[26] Zhang, L.-B.; Wang, D.-X.; Zhao, L.; Wang, M.-X. J. Org. Chem. 2012, 77, 5584 . 
[27] Matoishi, K.; Sano, A.; Imai, N.; Yamazaki, T.; Yokoyama, M.; Sugai, T.; Ohta, H. Tetrahedron: Asymmetry 1998, 9, 1097.

[28] (a) Chen, P.; Gao, M.; Wang, D.-X.; Zhao, L.; Wang, M.-X. Chem. Common. 2012, 48, 3482.

(b) Chen, P.; Gao, M.; Wang, D.-X.; Zhao, L.; Wang, M.-X. J. Org. Chem. 2012, 77, 4063.

[29] Ao, Y.-F.; Wang, D.-X.; Zhao, L.; Wang, M.-X. Chem. Asian J. $\mathbf{2 0 1 5}, 10,938$.

[30] (a) Kielbasinski, P.; Rachwalski, M.; Mikolajczyk, M.; Szyrej, M.;
Wieczorek, M. W.; Wijtmans, R.; Rutjes, F. P. J. T. Adv. Synth. Catal. 2007, 349,1387.

(b) Kielbasinski, P.; Rachwalski, M.; Kwiatkowska, M.; Mikolajczyk, M.; Wieczorek, M. W.; Szyrej, M.; Sieron, L.; Rutjes, F. P. J. T. Tetrahedron: Asymmetry 2007, 18, 2108.

[31] Fernandes, B. C. M.; Mateo, C.; Kiziak, C.; Chmura, A.; Wacker, J.; Rantwijk, F. V.; Stolz, A.; Sheldon, R. A. Adv. Synth. Catal. 2006, $348,2597$. 